\title{
Positron Emission Tomography
}

M.E. Juweid and O.S. Hoekstra, eds.

New York, NY: Humana Press, 2011, 364 pages, $\$ 129$

Positron Emission Tomography is a useful, informative book on PET methods in molecular biology in solid tumors authored by many well-known physicians and scientists from the United States and Europe. Besides ${ }^{18} \mathrm{~F}-\mathrm{FDG}$ PET, the book describes newer and promising PET radiotracers such as the precursors of amine metabolism, ${ }^{11} \mathrm{C}$ - or ${ }^{18} \mathrm{~F}-\mathrm{L}-3,4$-dihydroxyphenylalanine (L-DOPA) and ${ }^{11} \mathrm{C}-5$ hydroxy-L-tryptophan (5-HTP) for neuroendocrine tumors; $6-{ }^{18} \mathrm{~F}$-fluorodopamine is a highly specific agent for localization of adrenal and extraadrenal pheochromocytoma, and ${ }^{11} \mathrm{C}$-hydroxyl ephedrine concentrates in the sympathetic adrenergic nerve terminals, making it useful in pheochromocytoma and cardiac imaging. ${ }^{11} \mathrm{C}$-metomidate, an imidazole methyl ester, is a potent inhibitor of a key enzyme in the synthesis of adrenal cortisol and aldosterone. The somatostatin ligands DOTANOC or DOTATOC labeled with ${ }^{11} \mathrm{C},{ }^{18} \mathrm{~F},{ }^{64} \mathrm{Cu}$, or the preferred ${ }^{68} \mathrm{Ga}$ because of its more suitable radiophysical properties will help in imaging of somatostatin receptor-positive tumors. PET using ${ }^{18} \mathrm{~F}-3^{\prime}$ deoxy-3'fluorthymidine (FLT), a fluorinated thymidine analog that reflects the activity of thymidine kinase 1 , is used to monitor treatment response in breast cancer or androgen ablation therapy by showing marked reduction in ${ }^{18} \mathrm{~F}$-FLT accumulation in tumor. ${ }^{18} \mathrm{~F}$-fluoroestriol uptake in breast cancer correlates with estrogen receptor expression in primary and metastatic breast cancer. ${ }^{68} \mathrm{Ga}$-labeled $\mathrm{F}\left(\mathrm{ab}^{\prime}\right)$ trastuzumab can noninvasively identify breast cancer patients overexpressing HER2 and hence be more likely to respond to trastuzumab treatment. Angiogenesis inhibitors such as fluorinated RGD peptide target $\alpha_{\mathrm{v}} \beta_{3}$ integrin receptor imaging and can predict response to therapy. Imidazole derivative ${ }^{18} \mathrm{~F}$-misonidazole has potential for more efficient radiotherapy by increasing the dose delivered to the hypoxic fraction of the tumor tissue. ${ }^{11} \mathrm{C}$-acetate, ${ }^{11} \mathrm{C}$ - or ${ }^{18} \mathrm{~F}$-labeled choline, and ${ }^{18} \mathrm{~F}$-labeled 1 - $\left(2^{\prime}\right.$-deoxy $2^{\prime}$-fluoro- $\beta$-D-arabinofuranosyl (FMAU), a thymidine analog, appear promising in prostate cancer. ${ }^{18} \mathrm{~F}$-sodium fluoride is being increasingly applied for imaging of bone metastasis. This book goes beyond ${ }^{18} \mathrm{~F}$-FDG glucose metabolic imaging and describes imaging based on other metabolic processes, such as tumor cell proliferation, angiogenesis, and estrogen and Her2 neu receptor expression for treatment and management of cancer

COPYRIGHT @ 2012 by the Society of Nuclear Medicine, Inc.

Published online Apr. 27, 2012

DOI: 10.2967/jnumed.112.105403 patients. It reminds us how closely molecular imaging is based on body metabolism.

PET for follow-up and prognosis of residual lesions in testicular cancers and pitfalls in imaging mature teratoma are covered. Challenges in managing unresectable brain tumors, stereotactic radiosurgery, limitations of morphologic brain imaging in accurate tumor delineation, detection of anaplastic tumors, and specific challenges in pediatric brain tumors are covered. Labeled amino acid tracers such as ${ }^{11} \mathrm{C}$-methylL-methionine phenylalanine, $2-{ }^{18} \mathrm{~F}$-fluoro-L-tyrosine, and $2-{ }^{18} \mathrm{~F}-$ fluoro-L-phenylalanine show potential in the study of protein synthesis rate by active incorporation into proteins. Radiotracers that are not incorporated into proteins, such as $4-{ }^{18} \mathrm{~F}$-fluoro- $\alpha$-methyl-L-tyrosine $\left({ }^{18} \mathrm{~F}-\mathrm{FMT}\right), 3$,4-dihydroxy$6-{ }^{18} \mathrm{~F}-$ fluoro-L-phenylalanine (F-DOPA), and $O-\left(2-{ }^{18} \mathrm{~F}-\right.$ fluoroethyl)-L-tyrosine hold potential to evaluate the transport process. The usefulness of PET/CT in early and late response assessment in esophageal cancers, radiotherapy planning in head and neck cancers, posttherapy surveillance, and early detection of recurrence are covered. The limitations of ${ }^{18} \mathrm{~F}-\mathrm{FDG}$ imaging, cost and reimbursement issues, PET/CT effectiveness in recurrent lung cancer, and therapy monitoring cost-effectiveness are covered. ${ }^{18} \mathrm{~F}-\mathrm{FDG}$ quantification, SUVs, and imaging in breast cancer recurrence, in disease extent, and in response evaluation using PET are covered. A conclusion and summary in some chapters help reinforce important highlights in the chapter.

The 18 chapters are organized in a consistent format of introduction, applications in the particular malignancy, and usefulness in pretreatment staging, restaging, therapy monitoring, posttherapy surveillance, and other select indications such as evaluating the transformation of indolent lymphoma to an aggressive histology. Other diagnostic methods for staging of tumors, including relevant conventional crosssectional imaging methods, are briefly described. The chapter on PET/CT in radiotherapy in lung cancer helps us understand target volumes, autodelineation tools, and manual editing of target volumes used in the planning and administration of the most effective radiotherapy for maximal chance of long-term survival with acceptable side effects. Different subtypes of malignancies are described, and the inclusion of clinically relevant details such as tumor types, etiology, incidence and presentation, TNM staging, status of nodal disease, distant metastasis, prognosis of various tumor stages, and results of study trials involving PET are covered. 
Most chapters have illustrative images, whereas an occasional chapter is image-depleted. For example chapter 11, on sarcomas, can be improved by the inclusion of some images. The images are of good quality despite the nonglossy paper, and the fused PET/CT images in color allow for better appreciation of the PET abnormality in the framework of the adjacent morphologic structures.

Overall the book is informative and useful, although some chapters, such as the brain chapter, were heavy reading because of the many newer PET agents. This book is a good reference manual giving practical tips for goodquality images, covering false-positive and false-negative findings, and offering differential diagnosis for less certain PET findings. It gives information on the sensitivity, specificity, positive and negative predictive value, and accuracy rate of PET compared with other conventional imaging methods for tumors. The combined perspectives of the referring and interpreting physicians give depth to each chapter. As mentioned in the preface of this book, care of the oncologic patient involves a multidisciplinary team approach, and PET/CT is playing an expanding and important role in tumor management during several stages of a patient's malignancy. The PET findings can play an important role in upstaging or downstaging tumors, with consequent treatment implications. The book gives valuable tips on what to do or avoid during PET so as to produce good-quality images. Each chapter has adequate references, and some chapters have extensive references for additional reading.

Overall, this is a well-written book blending facts and data from the clinical and molecular imaging perspectives on solid malignancies. I highly recommend the book as a useful reference for physicians involved in PET and its interpretation. The book gives an exciting glimpse of the newer radiotracers that are or will soon be available to advance molecular imaging from the present-day ${ }^{18} \mathrm{~F}-\mathrm{FDG}$ imaging based on the glucose metabolic pathway to imaging based on other metabolic pathways. The book may also appeal to oncologists and surgeons who refer patients for PET.

\section{Usha A. Joseph}

University of Texas Medical School at Houston

6431 Fannin, Suite 2.130b

Houston, TX 77030

E-mail: usha.a.joseph@uth.tmc.edu

\section{Erratum}

In abstract 1159 of the 2012 abstract book $\left({ }^{64} \mathrm{Cu}\right.$ DOTA-trastuzumab $\left[{ }^{64} \mathrm{Cu}-\mathrm{Herceptin}\right] / \mathrm{PET}$ effectively visualizes metastatic breast cancer in HER2-positive patients. J Nucl Med. 2012;53[suppl 1]:229P), 2 names were omitted from the author list. The authors and affiliations should read as follows: "J.R. Bading, S. Tong, J. Reyes, M. Carroll, E. Poku, J.K. Miles, D. Colcher, J.E. Shively, J.Y. Wong, A. Raubitschek, J.E. Mortimer, City of Hope, Duarte, CA; P.S. Conti, University of Southern California, Los Angeles CA." 